KSUPT-03/1 January 2003

\title{
Galactic Foreground Constraints from the Python V Cosmic Microwave Background Anisotropy Data
}

\author{
Pia Mukherjee ${ }^{1,2}$, Kim Coble $^{3,4}$, Mark Dragovan $^{5}$, Ken Ganga $^{6}$, John Kovac ${ }^{3}$, Bharat Ratra ${ }^{1}$, and \\ Tarun Souradeep ${ }^{1,7}$
}

\begin{abstract}
We constrain Galactic foreground contamination of the Python V cosmic microwave background anisotropy data by cross correlating it with foreground contaminant emission templates. To model foreground emission we use 100 and $12 \mu \mathrm{m}$ dust emission templates and two point source templates based on the PMN survey. The analysis takes account of inter-modulation correlations in 8 modulations of the data that are sensitive to a large range of angular scales and also densely sample a large area of sky. As a consequence the analysis here is highly constraining. We find little evidence for foreground contamination in an analysis of the whole data set. However, there is indication that foregrounds are present in the data from the larger-angular-scale modulations of those Python V fields that overlap the region scanned earlier by the UCSB South Pole 1994 experiment. This is an independent consistency cross-check of findings from the South Pole 1994 data.
\end{abstract}

Subject headings: cosmology: observation — cosmic microwave background — diffuse radiation — dust, extinction

\section{Introduction}

While cosmic microwave background (CMB) anisotropy data have started to provide interesting constraints on cosmological parameters (see, e.g., Podariu et al. 2001; Page 2002; Mukherjee et

\footnotetext{
${ }^{1}$ Department of Physics, Kansas State University, 116 Cardwell Hall, Manhattan, KS 66506.

${ }^{2}$ Present address: Department of Physics and Astronomy, University of Oklahoma, 440 W. Brooks Street, Norman, OK 73019.

${ }^{3}$ Enrico Fermi Institute, University of Chicago, 5640 S. Ellis Ave., Chicago, IL 60637.

${ }^{4}$ Adler Planetarium and Astronomy Museum, 1300 S. Lake Shore Dr., Chicago, IL 60605.

${ }^{5}$ Jet Propulsion Laboratory, California Institute of Technology, 4800 Oak Grove Drive, 169-506, Pasadena, CA 91109.

${ }^{6}$ Infrared Processing and Analysis Center, California Institute of Technology, 100-22, Pasadena, CA 91125.

${ }^{7}$ Permanent address: IUCAA, Post Bag 4, Ganeshkhind, Pune 411007, India.
} 
al. 2002b, 2002c; Benoît et al. 2002; Ruhl et al. 2002; Kuo et al. 2002; Slosar et al. 2002 for recent results, and Peebles \& Ratra 2003 for a review), Galactic emission foreground contaminants in them are still not well understood. Robust constraints on cosmological parameters from these data require a better understanding of the effect of these contaminants.

In this paper we study foreground contaminants in the Python V (hereafter PyV) CMB anisotropy data. Python $\mathrm{V}$ is the latest of the Python experiments at the South Pole. Coble et al. (1999, 2003) describe the PyV experiment, observations, and data reduction. Dragovan et al. (1994), Ruhl et al. (1995), and Platt et al. (1997) describe Python I-III and Rocha et al. (1999) and Mukherjee et al. (2002c) derive constraints on cosmological parameters from these data. Coble et al. (2003) also describe the procedure used to create maps of the sky with PyV and Python III data; these maps were compared to infer consistency and indirectly deduce the lack of significant foreground contamination in these data.

The PyV data are acquired at a frequency of $\sim 40 \mathrm{GHz}$. Two regions of sky covering $598 \mathrm{deg}^{2}$ in the southern hemisphere were observed (the "main PyV" region and a smaller region, the fields labelled 'sa', 'sb', and 'sc' in Coble et al. 2003, that encompasses the region scanned earlier by the UCSB South Pole 1994 experiment - hereafter the "SP94 overlap" region). 690 fields were scanned in all (345 effective fields were scanned with 2 detector feeds separated by 2.8 in azimuth on the sky) with an asymmetric Gaussian beam of FWHM $0.94 \times 1.02$. Once the telescope was positioned on each field, the chopper smoothly scanned in azimuth with a throw of $17^{\circ}, 128$ samples were recorded in each chopper cycle, and 164 chopper cycles of data were taken of a given field. The densely sampled data were then modulated in software using the first eight cosine harmonics of the chopper cycle (hereafter modulations - or in Tables mods - 1-8). The modulations approach used has the advantage of filtering out some of the contaminants in the time stream, and also provides a rapid means of compressing a large amount of data into a more manageable size. All the modulations, other than the first, were apodized by a Hann window to reduce ringing in multipole space and down weight data taken during chopper turnaround. The resulting data are sensitive to angular scales corresponding to multipole moments ranging from $l \sim 40$ to $l \sim 260$. More details about the particular observing strategy employed and results found, such as the angular power spectrum of the data, may be found in Coble et al. (2003); see Souradeep \& Ratra (2001) for details about the window functions.

For this foregrounds analysis, we follow the general method outlined for example in Hamilton \& Ganga (2001) and Mukherjee et al. (2002a), doing a multi-modulation analysis here rather than the multi-(frequency) channel analysis discussed in these papers. In doing so we extend the preliminary estimate presented in Coble et al. (1999). Here we use the technique of Souradeep \& Ratra (2001) to account for correlations between modulations, in data that are sensitive to a substantial range of angular scales, as well as compare to what has been found from the SP94 experiment (Gundersen et al. 1995; Ganga et al. 1997) data about foreground contamination in part of the PyV region (Hamilton \& Ganga 2001; Mukherjee et al. 2002a). 
CMB data have previously been correlated with foreground templates (DMR: Kogut et al 1996a, 1996b; 19GHz: de Oliveira-Costa et al. 1998; Saskatoon: de Oliveira-Costa et al. 1997; OVRO: Leitch et al. 1997, Mukherjee et al. 2002a; SP94: Hamilton \& Ganga 2001, Mukherjee et al. 2002a; Tenerife: de Oliveira-Costa et al. 1999, 2002, Mukherjee et al. 2001; QMAP: de Oliveira-Costa et al. 2000; MAX: Lim et al. 1996, Ganga et al. 1998; and Boomerang: Masi et al. 2001 $)^{8}$. Correlations between CMB data and infra-red emission seem to be roughly consistent with free-free emission, spectrally, over a wide range of frequencies (10 to $90 \mathrm{GHz}$ ) and angular scales $\left(7^{\circ}\right.$ to $\left.7^{\prime}\right)$, with some evidence for a contribution from spinning dust emission with a peak around 15 to $20 \mathrm{GHz}$. In general though the contamination from foregrounds has not been found to be large in any experiment, but on the detail level residual foregrounds can cause problems with parameter estimation and non-Gaussianity tests for high precision CMB data. ${ }^{9}$

To model diffuse Galactic emission, we use the $100 \mu \mathrm{m}$ IRAS+DIRBE map (Schlegel, Finkbeiner, \& Davis 1998) as a tracer of thermal emission from interstellar dust, and the $12 \mu \mathrm{m}$ map (D. Finkbeiner, private communication, 2000) as a tracer of emission from ultra-small dust grains. Emission from small dust grains is still under study (Finkbeiner et al. 2002). Such grains may contribute significantly at microwave frequencies according to a model by Draine \& Lazarian (1998a,b), and this has been reviewed from the CMB data point of view by Kogut (1999) and Draine \& Lazarian (1999). The Haslam $408 \mathrm{MHz}$ map (Haslam et al. 1981) of synchrotron emission was not used as it does not have enough resolution for all the modulations of the PyV experiment to be simulated on it. At the same time synchrotron emission is not likely to contribute significantly at $40 \mathrm{GHz}$. We have tried to use the SHASSA $H_{\alpha}$ data (Gaustad et al. 2001) as a tracer of free-free emission, but found that the template has insufficient resolution for our purpose.

We also use two point source templates created from the PMN survey (Wright et al. 1994). The one called PMN has been converted to $\delta T_{C M B}$ (equivalent temperature fluctuations in the $\mathrm{CMB}$ at $40 \mathrm{GHz}$ ) using the spectral indices given in the survey, while the one called PMN0 is converted to a flux at $40 \mathrm{GHz}$ assuming a flat spectrum extrapolated from the flux measurement at $4.85 \mathrm{GHz}$. The assumption of a flat spectrum is conservative in that it is likely to overestimate the flux at $40 \mathrm{GHz}$. Neither case is correct, since spectral indices have not been measured for all of the sources, in which case a flat spectrum is assumed. The two cases cover a reasonable range

\footnotetext{
${ }^{8}$ DASI (Halverson et al. 2002) and VSA (Taylor et al. 2002) are interferometric experiments, that observed at frequencies of $26-36 \mathrm{GHz}$ and are sensitive to multipoles of $\sim 100$ to 900 . Point sources are the dominant source of contamination and the effect of contamination from diffuse Galactic foregrounds upon the CMB power spectrum is inferred to be small for both data sets. A full cross-correlation analysis of VSA data is underway (Dickinson et al., in preparation).

${ }^{9}$ The CMB anisotropy is often thought to have been generated by quantum mechanical fluctuations in a weakly coupled scalar field during an early epoch of inflation and thus would be a realization of a spatially stationary Gaussian random process (see, e.g., Ratra 1985; Fischler, Ratra, \& Susskind 1985). Measurements lend fairly strong support to this Gaussianity assumption (see, e.g., Park et al. 2001; Shandarin et al. 2002; Santos et al. 2002; Polenta et al. 2002). See Park, Park, \& Ratra (2002) for the effects of foreground contamination on Gaussianity tests based on anticipated MAP data.
} 
of possibilities. These templates were also used in the preliminary foreground analysis of Coble et al. (1999).

Each of the Galactic emission maps have been converted into a template for cross correlation with the PyV data by simulating the PyV observing strategy on it, taking account of the asymmetric beam. Since a chopper synchronous offset and a ground shield offset were removed from the data, we account for this by adding the chopper synchronous offset and ground shield constraint matrices to the noise matrix and marginalizing over them in the analysis. The CMB theory covariance matrix is modelled using a spatially-flat cosmological constant dominated CDM model with non-relativistic matter density parameter $\Omega_{0}=0.3$, scaled baryonic matter density parameter $\Omega_{B} h^{2}=0.021$ (here $h$ is the present value of the Hubble parameter in units of $100 \mathrm{~km} \mathrm{~s}^{-1} \mathrm{Mpc}^{-1}$ ), and age $t_{0}=14$ Gyr (Ratra et al. 1999a) with the CMB anisotropy normalized to a quadrupole moment amplitude $Q_{\text {rms-PS }}=20 \mu \mathrm{K}$.

\section{Correlations}

\subsection{Method}

We follow the general method outlined for example in Hamilton \& Ganga (2001) and Mukherjee et al. (2002a), doing a multi-modulation analysis here rather than the multi-(frequency) channel

analysis discussed in these papers. The method assumes that the data are a linear combination of CMB anisotropies and foreground components,

$$
y=a X+x_{\mathrm{CMB}}+n .
$$

Here $y$ is a $n_{\text {mod }} \times N$ element vector containing the data, $n$ is the corresponding noise vector, $x_{\mathrm{CMB}}$ is the CMB signal, and $X$ is a $\left(n_{\bmod } \times N\right) \times n_{\text {mod }}$ element matrix containing the simulated foreground template, $N$ being the number of data points per modulation and $n_{\text {mod }}$ being the number of modulations. In a given column $X$ contains mostly zeros except for the rows corresponding to that modulation, where it contains the simulated template signal. The vector a contains $n_{\bmod }$ elements that represent the amplitude of the correlated foreground signal. If the noise and CMB anisotropies are uncorrelated Gaussian distributed variables with zero mean, minimizing $\chi^{2}$ leads to a best fit estimate of

$$
\hat{a}=\left[X^{T} C^{-1} X\right]^{-1} X^{T} C^{-1} y,
$$

where $C$ is the $\left(n_{\bmod } \times N\right) \times\left(n_{\bmod } \times N\right)$ element total covariance matrix (sum of the theory covariance matrix that models the CMB signal, the noise covariance matrix, and any constraint matrices). The vector $\hat{a}$ then contains the best estimate for the correlation slopes for the corresponding template for all modulations, given all information about inter-modulation correlations. The matrix

$$
\Sigma=\left\langle\hat{a}^{2}\right\rangle-\langle\hat{a}\rangle^{2}=\left[X^{T} C^{-1} X\right]^{-1}
$$


is its covariance matrix. The rms amplitudes of temperature fluctuations in the data that results from the correlation is $\Delta T=(\hat{a} \pm \delta \hat{a}) \sigma_{\text {fore }}$, where $\sigma_{\text {fore }}$ contains the rms deviations of the corresponding foreground template in the different modulations.

The method assumes that our foreground emission maps are good enough to model the foregrounds in the data accurately in all the different modulations, at a frequency different from that of the original foreground emission map, and that eq. (1) explains all the structure in all modulations of the data. If we further assume that the ratio of the signal in the data and that in the foreground template is the same for all the modulations, then a net correlation slope can be found using

$$
\bar{a}=\frac{\operatorname{Total}\left(\Sigma^{-1} \mathrm{a}\right)}{\operatorname{Total}\left(\Sigma^{-1}\right)}
$$

Here Total denotes the sum of all elements of a matrix or vector. This is thus a weighted average taking account of correlations between the $a$ values of the different modulations. We may choose not to make the last assumption (see discussion in $\S 2.3$ ).

\section{2. $\quad$ Results}

The correlation slopes obtained from a complete inter-modulation analysis, using all the PyV data points, for each foreground emission template individually, are given in Table 1 (top panel). We do not find any significant correlation. The net correlation slopes (i.e., the weighted mean of the correlation slopes given in the table, taking cross-modulation correlations into account) are not significant, with one standard deviation upper limits of $20 \mu \mathrm{K}(\mathrm{MJy} / \mathrm{sr})^{-1}, 115 \mu \mathrm{K}(\mathrm{MJy} / \mathrm{sr})^{-1}, 93$ $\mu \mathrm{K} / \mu \mathrm{K}$, and $344 \mu \mathrm{K}(\mathrm{MJy} / \mathrm{sr})^{-1}$, for the $100 \mu \mathrm{m}$, the $12 \mu \mathrm{m}$, the PMN, and the PMN0 templates, respectively. These limits on $100 \mu \mathrm{m}$ and PMN0 correlation slopes compare well with those of Table 2 of Coble et al. (1999). (There are some errors in the PMN correlation slopes of Table 2 and the rms values in Table 3 of Coble et al. 1999.) The upper limits obtained from using all modulations of the data together are given in Table 3 .

The results of repeating this analysis for just the SP94 overlap region are also given in Table 1 (bottom panel). ${ }^{10}$ The uncertainties in the estimated correlation slopes are higher here, with the number of data points down by a factor of almost 8 (the time spent observing this region was less than 1:8 of the total observing time), but the level of associated temperature fluctuations are relatively higher, at least for the $100 \mu \mathrm{m}$ template. As seen from Tables 2 and 3, the rms of the data and the $100 \mu \mathrm{m}$ template are higher in this patch of sky. Again, no significant correlations are found. The 100 and $12 \mu \mathrm{m}$ templates and the PMN and PMN0 templates have significant (weighted) correlation coefficients in this region of sky, with the correlation reducing somewhat

\footnotetext{
${ }^{10}$ An outlier that affects 6 data points per feed per modulation is seen in the $12 \mu \mathrm{m}$ template, hence these data points are ignored whenever this region is being analysed. Outliers are harder to pick out over larger regions of sky but they also affect the result less, so nothing is removed when analysing the full data set.
} 
with increasing modulation. (These templates are much less like each other when all the PyV fields are considered.) Yet when all modulations of the data are analysed together, joint template fits do not detect any correlations. The net correlation slopes obtained from a complete inter-modulation analysis of the SP94 overlap region are not significant, with $1 \sigma$ upper limits of $29 \mu \mathrm{K}(\mathrm{MJy} / \mathrm{sr})^{-1}$, $524 \mu \mathrm{K}(\mathrm{MJy} / \mathrm{sr})^{-1}, 259 \mu \mathrm{K} / \mu \mathrm{K}$, and $1551 \mu \mathrm{K}(\mathrm{MJy} / \mathrm{sr})^{-1}$, for the $100 \mu \mathrm{m}$, the $12 \mu \mathrm{m}$, the PMN, and the PMN0 templates, respectively. The upper limits obtained from using all modulations of the data together are given in Table 3.

\subsection{Low $l$ Modulations}

As seen from Table 2, the signal in the foreground templates falls more steeply with increasing modulation number than does the data. So the case here, of fitting for several modulations simultaneously, is somewhat different from the case of fitting multi-frequency data: if a certain foreground is present in the data, picking it out in the higher modulations will be harder. But the cumulative effect of the other modulations is significant on the estimated correlation slope for any modulation; Figure 2 of Coble et al. (1999) shows how much the CMB signal in different modulations overlap. Hence simultaneously fitting for the correlation slopes in all the modulations of the data may not be the most appropriate thing to do. It is also important to note that the foregrounds may not have been accurately modelled in the higher modulations because of insufficient resolution (given the dense sampling in the data). There may also be different kinds of unmodelled or incorrectly modelled foregrounds/errors in different modulations. Hence it is useful to also look at the results from analysing say the first three modulations together, because across these modulations the data and the foreground signal seem to be roughly similar as regards rms values, or each modulation individually, at the cost of increased uncertainty in the estimates.

If we look at only the first modulation in the SP94 overlap region, we find a $1.8 \sigma$ correlation slope of $80 \pm 45 \mu \mathrm{K}(\mathrm{MJy} / \mathrm{sr})^{-1}(36 \pm 21 \mu \mathrm{K})$ for the $100 \mu \mathrm{m}$ template (Table 4$)$. For the $12 \mu \mathrm{m}$ template the correlation found is just less than a sigma, but here the two dust templates have a weighted correlation coefficient of 0.65 , and performing a joint correlation of the data with these two templates results in a $1.9 \sigma$ correlation slope of $115 \pm 60 \mu \mathrm{K}(\mathrm{MJy} / \mathrm{sr})^{-1}(52 \pm 27 \mu \mathrm{K})$ for the $100 \mu \mathrm{m}$ template at the cost of a $1 \sigma$ negative correlation with the $12 \mu \mathrm{m}$ template. This may be the signal found in the foregrounds analysis of the SP94 data (Hamilton \& Ganga 2001; Mukherjee et al. 2002a; for earlier qualitative indications see Ganga et al. 1997; Ratra et al. 1999b). While there a detection was aided by the presence of 7 frequency channels, here the error bars for the individual modulation correlations are large. This could be just a chance correlation on the other hand, and the probability of that is reflected in the significance of the result. It might also be relevant to note here that the SP94 data was only one dimensional while here the PyV 'sa', 'sb', and 'sc' data are essentially two dimensional.

If we look at only the second modulation by itself, the correlation slopes are as shown in Table 4 , and even though the correlation coefficient between the 12 and $100 \mu \mathrm{m}$ templates is 0.70 nothing 
is gained by a joint fit this time.

In the first two modulations together some correlation with the $100 \mu \mathrm{m}$ template is detected consistent with the above estimates. When the first three modulations are correlated simultaneously, a $1.6 \sigma$ correlation slope of $58 \pm 36 \mu \mathrm{K}(\mathrm{MJy} / \mathrm{sr})^{-1}(25 \pm 16 \mu \mathrm{K})$ is found with the $100 \mu \mathrm{m}$ template in the first modulation and a $1 \sigma 710 \pm 695 \mu \mathrm{K}(\mathrm{MJy} / \mathrm{sr})^{-1}(14 \pm 14 \mu \mathrm{K})$ correlation is found with the $12 \mu \mathrm{m}$ template in the second modulation. The correlation coefficient between the templates is high and correlations of similar significance are found in the same modulations when the two templates are analysed jointly.

Regarding the point source templates, nothing significant shows up in an individual template analysis (Table 4). When analysing the first two modulations together the two point source templates have a correlation coefficient of 0.8 and a correlation slope of $6555 \pm 4994 \mu \mathrm{K}(\mathrm{MJy} / \mathrm{sr})^{-1}$ $(28 \pm 21 \mu \mathrm{K})$ at $1.3 \sigma$ is found with the PMN0 template in modulation 1 at the cost of a $1 \sigma$ negative correlation with the PMN template in the same modulation. When analysing the first three modulations together the point source templates have a high correlation coefficient and a 1.2 $\sigma$ correlation of $5625 \pm 4498 \mu \mathrm{K}(\mathrm{MJy} / \mathrm{sr})^{-1}(24 \pm 19 \mu \mathrm{K})$ is found with the PMN0 template in modulation 1 at the cost of a $-1 \sigma$ correlation with the PMN template in the same modulation.

When all the PyV fields are taken together (690 in each modulation), a $1 \sigma$ correlation with the $100 \mu \mathrm{m}$ template shows up in the first modulation (when the first modulation is analysed by itself, or jointly with the second, or jointly with the second and third), and a $1 \sigma$ correlation with the PMN0 template shows up in the first modulation when it is analysed together with the second modulation and jointly with the PMN template. The uncertainty in the correlation slopes with the point source templates is lowest in the third and fourth modulations, but nothing significant shows up when these modulations are analysed separately. Hence consistent correlations (in the low-l modulations and at low significance) are seen even when both the PyV regions are analysed together.

\subsection{Summary}

When all the modulations of the data are analysed together, no significant correlations are found, in the whole PyV data set or in the SP94 overlap region. These results are summarized in Table 3 and plotted in Figure 1.

We have discussed the motivations for looking at individual modulations separately, and we find some indication of foregrounds in the low $l$ modulations. Correlation slopes for some of these low $l$ modulations are summarized in Table 4 . We see that greater than $1 \sigma$ correlations show up in the SP94 overlap region with the $100 \mu \mathrm{m}$ template in particular, but also with the $12 \mu \mathrm{m}$ template and the PMN0 template in some modulations in a joint modulation analysis of a few modulations. And consistent correlations are seen when all the PyV data fields are analysed together. 
According to the foreground model discussed for example in Mukherjee et al. (2002a), if the $100 \mu \mathrm{m}$ correlations are from both free-free emission and spinning dust emission, then by $40 \mathrm{GHz}$ the contribution from spinning dust emission (as traced by the $12 \mu \mathrm{m}$ template) may have again dropped below that from free-free (the frequency at which spinning dust emission peaks depends on the details of the spinning dust emission model and this can also vary from region to region), so that we expect $100 \mu \mathrm{m}$ correlations to be more significant than $12 \mu \mathrm{m}$ correlations at this frequency (see Figure 1a). Although given the error bars (the numbers in Table 4), we do not expect to see significant correlations in the PyV data. These imply that what is found here is not inconsistent with the foreground model that other CMB data sets tentatively seem to point to (depicted in Figure 1).

\section{Conclusion}

Using the method of cross correlating CMB anisotropy data with foreground contaminant emission templates, we find little evidence for foreground contamination in the whole PyV region when analysing all modulations of the data together. There is however indication of foreground contamination in the low- $l$ modulations of the $\mathrm{PyV}$ fields that cover the region scanned earlier by the SP94 experiment, consistent with results from the SP94 data. This is a valuable test, indicating consistency between results found using data from two different experiments. Given the uncertainties, our findings are not inconsistent with the two component dust-correlated (free-free and spinning dust) foreground emission model that other data sets tentatively seem to point to (Mukherjee et al. 2002a).

We thank D. Finkbeiner for the $12 \mu \mathrm{m}$ dust map. PM, BR, and TS acknowledge support from NSF CAREER grant AST-9875031. KC is supported by NSF grant AST-0104465. This work was partially carried out at the Infrared Processing and Analysis Center and the Jet Propulsion Laboratory of the California Institute of Technology, under a contract with the National Aeronautics and Space Administration. 
Table 1. Results of Correlating the PyV Data with Individual Foreground Emission Templates ${ }^{\mathrm{a}}$

\begin{tabular}{|c|c|c|c|c|c|c|c|c|}
\hline Template & $\bmod 1$ & $\bmod 2$ & $\bmod 3$ & $\bmod 4$ & $\bmod 5$ & $\bmod 6$ & $\bmod 7$ & $\bmod 8$ \\
\hline \multirow[t]{2}{*}{$100 \mu \mathrm{m}$} & $1 \pm 20$ & $-4 \pm 20$ & $-20 \pm 20$ & $-6 \pm 23$ & $5 \pm 30$ & $8 \pm 52$ & $-34 \pm 110$ & $-172 \pm 245$ \\
\hline & $0 \pm 4$ & $-1 \pm 3$ & $-2 \pm 2$ & $0 \pm 2$ & $0 \pm 1$ & $0 \pm 1$ & $0 \pm 1$ & $-1 \pm 1$ \\
\hline \multirow[t]{2}{*}{$12 \mu \mathrm{m}$} & $-82 \pm 131$ & $-75 \pm 123$ & $-79 \pm 120$ & $-133 \pm 126$ & $-257 \pm 140$ & $-372 \pm 182$ & $-228 \pm 259$ & $-476 \pm 516$ \\
\hline & $-2 \pm 3$ & $-2 \pm 3$ & $-1 \pm 2$ & $-2 \pm 2$ & $-3 \pm 1$ & $-3 \pm 1$ & $-1 \pm 1$ & $-1 \pm 1$ \\
\hline \multirow[t]{2}{*}{ PMN } & $137 \pm 123$ & $66 \pm 108$ & $-14 \pm 103$ & $-68 \pm 100$ & $-158 \pm 105$ & $-173 \pm 126$ & $-137 \pm 177$ & $8 \pm 252$ \\
\hline & $3 \pm 3$ & $2 \pm 3$ & $0 \pm 2$ & $-1 \pm 2$ & $-2 \pm 1$ & $-2 \pm 1$ & $-1 \pm 1$ & $0 \pm 1$ \\
\hline \multirow[t]{2}{*}{ PMN0 } & $684 \pm 502$ & $546 \pm 408$ & $237 \pm 382$ & $15 \pm 369$ & $-111 \pm 385$ & $-116 \pm 452$ & $-44 \pm 625$ & $972 \pm 986$ \\
\hline & $3 \pm 2$ & $3 \pm 2$ & $1 \pm 2$ & $0 \pm 1$ & $0 \pm 1$ & $0 \pm 1$ & $0 \pm 1$ & $1 \pm 1$ \\
\hline \multirow[t]{2}{*}{$100 \mu \mathrm{m}$} & $19 \pm 30$ & $18 \pm 30$ & $5 \pm 31$ & $12 \pm 35$ & $30 \pm 50$ & $92 \pm 94$ & $-104 \pm 266$ & $792 \pm 1051$ \\
\hline & $8 \pm 14$ & $6 \pm 11$ & $1 \pm 7$ & $2 \pm 6$ & $3 \pm 5$ & $4 \pm 4$ & $-2 \pm 4$ & $4 \pm 5$ \\
\hline \multirow[t]{2}{*}{$12 \mu \mathrm{m}$} & $116 \pm 570$ & $235 \pm 559$ & $-288 \pm 561$ & $-248 \pm 647$ & $-348 \pm 868$ & $-626 \pm 1672$ & $-2476 \pm 3871$ & $7207 \pm 11467$ \\
\hline & $2 \pm 12$ & $4 \pm 10$ & $-4 \pm 7$ & $-2 \pm 6$ & $-2 \pm 6$ & $-2 \pm 5$ & $-3 \pm 5$ & $4 \pm 6$ \\
\hline \multirow[t]{2}{*}{ PMN } & $205 \pm 327$ & $98 \pm 291$ & $70 \pm 266$ & $5 \pm 276$ & $78 \pm 320$ & $-123 \pm 436$ & $319 \pm 801$ & $862 \pm 1717$ \\
\hline & $5 \pm 8$ & $3 \pm 8$ & $1 \pm 6$ & $0 \pm 4$ & $1 \pm 4$ & $-1 \pm 3$ & $1 \pm 4$ & $3 \pm 5$ \\
\hline \multirow[t]{2}{*}{ PMN0 } & $1871 \pm 2030$ & $947 \pm 1722$ & $636 \pm 1607$ & $267 \pm 1645$ & $409 \pm 1958$ & $-1496 \pm 2851$ & $3495 \pm 5538$ & $9599 \pm 11042$ \\
\hline & $8 \pm 8$ & $5 \pm 8$ & $2 \pm 6$ & $1 \pm 5$ & $1 \pm 4$ & $-2 \pm 4$ & $3 \pm 4$ & $5 \pm 6$ \\
\hline
\end{tabular}

${ }^{\text {a }}$ For each template the correlation slopes (in units of $\mu \mathrm{K}(\mathrm{MJy} / \mathrm{sr})^{-1}$ for the dust and the PMN0 templates and in units of $\mu \mathrm{K} / \mu \mathrm{K}$ for the PMN template) are given in the first row and the corresponding temperature fluctuations (in $\mu \mathrm{K}$ ) are given in the second row. In the top panel we have used all the data points, and in the bottom panel we use only the fields in the SP94 overlap region. Cross-modulation correlations are accounted for and the 8 modulations represent decreasing angular scales covering $l \sim 40$ to $\sim 260$. 
Table 2. Rms Values of the Signal in the PyV Data and the Templates ${ }^{\mathrm{a}}$

\begin{tabular}{lccccc}
\hline \hline Modulation & $\begin{array}{c}\text { Data } \\
\mu \mathrm{K}\end{array}$ & $\begin{array}{c}100 \mu \mathrm{m} \\
\mathrm{MJy} / \mathrm{sr}\end{array}$ & $\begin{array}{c}12 \mu \mathrm{m} \\
\mathrm{MJy} / \mathrm{sr}\end{array}$ & $\begin{array}{c}\mathrm{PMN} \\
\mu \mathrm{K}\end{array}$ & $\begin{array}{c}\text { PMN0 } \\
\mathrm{MJy} / \mathrm{sr}\end{array}$ \\
\hline 1 & 92.0 & 0.222 & 0.022 & 0.022 & 0.004 \\
2 & 95.1 & 0.170 & 0.022 & 0.027 & 0.006 \\
3 & 79.4 & 0.100 & 0.019 & 0.022 & 0.005 \\
4 & 77.0 & 0.078 & 0.015 & 0.017 & 0.004 \\
5 & 78.6 & 0.047 & 0.010 & 0.013 & 0.003 \\
6 & 77.4 & 0.023 & 0.007 & 0.010 & 0.002 \\
7 & 69.8 & 0.011 & 0.006 & 0.007 & 0.002 \\
8 & 65.0 & 0.006 & 0.003 & 0.005 & 0.001 \\
\hline 1 & 123.0 & 0.455 & 0.022 & 0.025 & 0.004 \\
2 & 129.9 & 0.356 & 0.018 & 0.028 & 0.005 \\
3 & 104.9 & 0.221 & 0.013 & 0.022 & 0.004 \\
4 & 111.1 & 0.180 & 0.010 & 0.016 & 0.003 \\
5 & 113.6 & 0.103 & 0.007 & 0.012 & 0.002 \\
6 & 111.5 & 0.042 & 0.003 & 0.008 & 0.001 \\
7 & 95.7 & 0.015 & 0.001 & 0.004 & 0.001 \\
8 & 89.33 & 0.005 & 0.001 & 0.003 & 0.0005 \\
\hline
\end{tabular}

aThe top panel is for the whole PyV region with 690 fields per modulation. The bottom panel is for the SP94 overlap region with 90 fields per modulation.

Table 3. Correlation Slopes ${ }^{\mathrm{a}}$

\begin{tabular}{|c|c|c|c|c|c|}
\hline Fields & Modulations & $\begin{array}{c}100 \mu \mathrm{m} \\
\mu \mathrm{K}(\mathrm{MJy} / \mathrm{sr})^{-1}\end{array}$ & $\begin{array}{c}12 \mu \mathrm{m} \\
\mu \mathrm{K}(\mathrm{MJy} / \mathrm{sr})^{-1}\end{array}$ & $\begin{array}{c}\mathrm{PMN} \\
\mu \mathrm{K} / \mu \mathrm{K}\end{array}$ & $\begin{array}{c}\text { PMN0 } \\
\mu \mathrm{K}(\mathrm{MJy} / \mathrm{sr})^{-1}\end{array}$ \\
\hline all & all & 20 & 115 & 93 & 344 \\
\hline SP94 overlap & all & 29 & 524 & 259 & 1551 \\
\hline
\end{tabular}

${ }^{a}$ One standard deviation upper limits. 
Table 4. Correlation Slopes (or $1 \sigma$ Upper Limits) for Low $l$ Modulations

\begin{tabular}{lccccc}
\hline \hline Fields & Modulations & $\begin{array}{c}100 \mu \mathrm{m} \\
\mu \mathrm{K}(\mathrm{MJy} / \mathrm{sr})^{-1}\end{array}$ & $\begin{array}{c}12 \mu \mathrm{m} \\
\mu \mathrm{K}(\mathrm{MJy} / \mathrm{sr})^{-1}\end{array}$ & $\begin{array}{c}\mathrm{PMN} \\
\mu \mathrm{K} / \mu \mathrm{K}\end{array}$ & $\begin{array}{c}\mu \mathrm{K}(\mathrm{MJy} / \mathrm{sr})^{-1} \\
\text { all }\end{array}$ \\
1st 3 mods & 22 & 141 & 131 & 553 \\
all & 1st 2 mods & 24 & 162 & 154 & 658 \\
all & 1st mod & $29 \pm 29$ & 223 & 213 & 903 \\
\hline SP94 overlap & 1st 3 mods & $33 \pm 32$ & 590 & 332 & 1924 \\
SP94 overlap & 1st 2 mods & $50 \pm 36$ & 674 & 387 & 2215 \\
SP94 overlap & 1st mod & $80 \pm 45$ & 875 & 563 & 3401 \\
SP94 overlap & 2nd mod & $49 \pm 47$ & 889 & 473 & 2805 \\
\hline
\end{tabular}




\section{REFERENCES}

Benoît, A., et al. 2002, astro-ph/0210306

Coble, K., et al. 1999, ApJ, 519, L5

Coble, K., Dodelson, S., Dragovan, M., Ganga, K., Knox, L., Kovac, J., Ratra, B., \& Souradeep, T. 2003, ApJ, in press, astro-ph/0112506

de Oliveira-Costa, A., Kogut, A., Devlin, M. J., Netterfield, C. B., Page, L. A., \& Wollack, E. J. 1997, ApJ, 482, L17

de Oliveira-Costa, A., et al. 2000, ApJ, 542, L5

de Oliveira-Costa, A., et al. 2002, ApJ, 567, 363

de Oliveira-Costa, A., Tegmark, M., Gutierrez, C. M., Jones, A. W., Davies, R. D., Lasenby, A. N., Rebolo, R., \& Watson, R. A. 1999, ApJ, 527, L9

de Oliveira-Costa, A., Tegmark, M., Page, L. A., \& Boughn, S. P. 1998, ApJ, 509, L9

Dragovan, M., Ruhl, J. E., Novak, G., Platt, S. R., Crone, B., Pernic, R., \& Peterson, J. B. 1994 ApJ, 427, L67

Draine, B. T., \& Lazarian, A. 1998a, ApJ, 494, L19

Draine, B. T., \& Lazarian, A. 1998b, ApJ, 508, 157

Draine, B. T., \& Lazarian, A. 1999, in ASP Conf. Ser. 181, Microwave Foregrounds, ed. A. de Oliveira-Costa \& M. Tegmark (San Francisco: ASP), 133

Finkbeiner, D. P., Schlegel, D. J., Frank, C., \& Heiles, C. 2002, ApJ, 566, 898

Fischler, W., Ratra, B., \& Susskind, L. 1985, Nucl. Phys. B, 259, 730

Ganga, K., Ratra, B., Gundersen, J. O., \& Sugiyama, N. 1997, ApJ, 484, 7

Ganga, K., Ratra, B., Lim, M. A., Sugiyama, N., \& Tanaka, S. T. 1998, ApJS, 114, 165

Gaustad, J. E., McCullough, P. R., Rosing, W., \& Van Buren, D. 2001, PASP, 113, 1326

Gundersen, J. O., et al. 1995, ApJ, 443, L57

Halverson, N. W., et al. 2002, ApJ, 568, 38

Hamilton, J.-Ch., \& Ganga K.M. 2001, A\&A, 368, 760

Haslam, C. G. T., Klein, U., Salter, C. J., Stoffel, H., Wilson, W. E., Cleary, M. N., Cooke, D. J., \& Thomasson, P. 1981, A\&A, 100, 209 
Kogut, A. 1999, in ASP Conf. Ser. 181, Microwave Foregrounds, ed. A. de Oliveira-Costa \& M. Tegmark (San Francisco: ASP), 91

Kogut, A., Banday, A. J., Bennett, C. L., Górski, K. M., Hinshaw, G., \& Reach, W. T. 1996a, ApJ, 460, 1

Kogut, A., Banday, A. J., Bennett, C. L., Górski, K. M., Hinshaw, G., Smoot, G. F., \& Wright, E. L. 1996b, ApJ, 464, L5

Kuo, C. L., et al. 2002, astro-ph/0212289

Leitch, E. M., Readhead, A. C. S., Pearson, T. J., \& Myers, S. T. 1997, ApJ, 486, L23

Lim, M. A., et al. 1996, ApJ, 469, L69

Masi, S., et al. 2001, ApJ, 553, 93

Mukherjee, P., Dennison, B., Ratra, B., Simonetti, J. H., Ganga, K., \& Hamilton, J.-Ch. 2002a, ApJ, 579, 83

Mukherjee, P., Ganga, K., Ratra, B., Rocha, G., Souradeep, T., Sugiyama, N., \& Górski, K. M. 2002c, astro-ph/0209567

Mukherjee, P., Jones, A. W., Kneissl, R., \& Lasenby, A. N. 2001, MNRAS, 320, 224

Mukherjee, P., Souradeep, T., Ratra, B., Sugiyama, N., \& Górski, K. M. 2002b, astro-ph/0208216

Page, L. 2002, astro-ph/0202145

Park, C.-G., Park, C., \& Ratra, B. 2002, ApJ, 568, 9

Park, C.-G., Park, C., Ratra, B., \& Tegmark, M. 2001, ApJ, 556, 582

Peebles, P. J. E., \& Ratra, B. 2003, Rev. Mod. Phys, in press, astro-ph/0207347

Platt, S. R., Kovac, J., Dragovan, M., Peterson, J. B., \& Ruhl, J. E. 1997, ApJ, 475, L1

Podariu, S., Souradeep, T., Gott, J. R., Ratra, B., \& Vogeley, M. S. 2001, ApJ, 559, 9

Polenta, G., et al. 2002, ApJ, 572, L27

Ratra, B. 1985, Phys. Rev. D, 31, 1931

Ratra, B., Ganga, K., Stompor, R., Sugiyama, N., de Bernardis, P., \& Górski, K. M. 1999a, ApJ, 510,11

Ratra, B., Stompor, R., Ganga, K., Rocha, G., Sugiyama, N., \& Górski, K. M. 1999b, ApJ, 517, 549 
Rocha, G., Stompor, R., Ganga, K., Ratra, B., Platt, S. R., Sugiyama, N., \& Górski, K. M. 1999, ApJ, 525, 1

Ruhl, J. E., et al. 2002, astro-ph/0212229

Ruhl, J. E., Dragovan, M., Platt, S. R., Kovac, J., \& Novak, G. 1995, ApJ, 453, L1

Santos, M. G., et al. 2002, Phys. Rev. Lett., 88, 241302

Schlegel, D. J., Finkbeiner, D. P., \& Davis, M. 1998, ApJ, 500, 525

Shandarin, S. F., Feldman, H. A., Xu, Y., \& Tegmark, M. 2002, ApJS, 141, 1

Slosar, A., et al. 2002, astro-ph/0212497

Souradeep, T., \& Ratra, B. 2001, ApJ, 560, 28

Taylor, A. C., et al. 2002, MNRAS, submitted, astro-ph/0205381

Wright, A. E., Griffith, M. R., Burke, B. F., \& Ekers, R. D. 1994, ApJS, 91, 111 


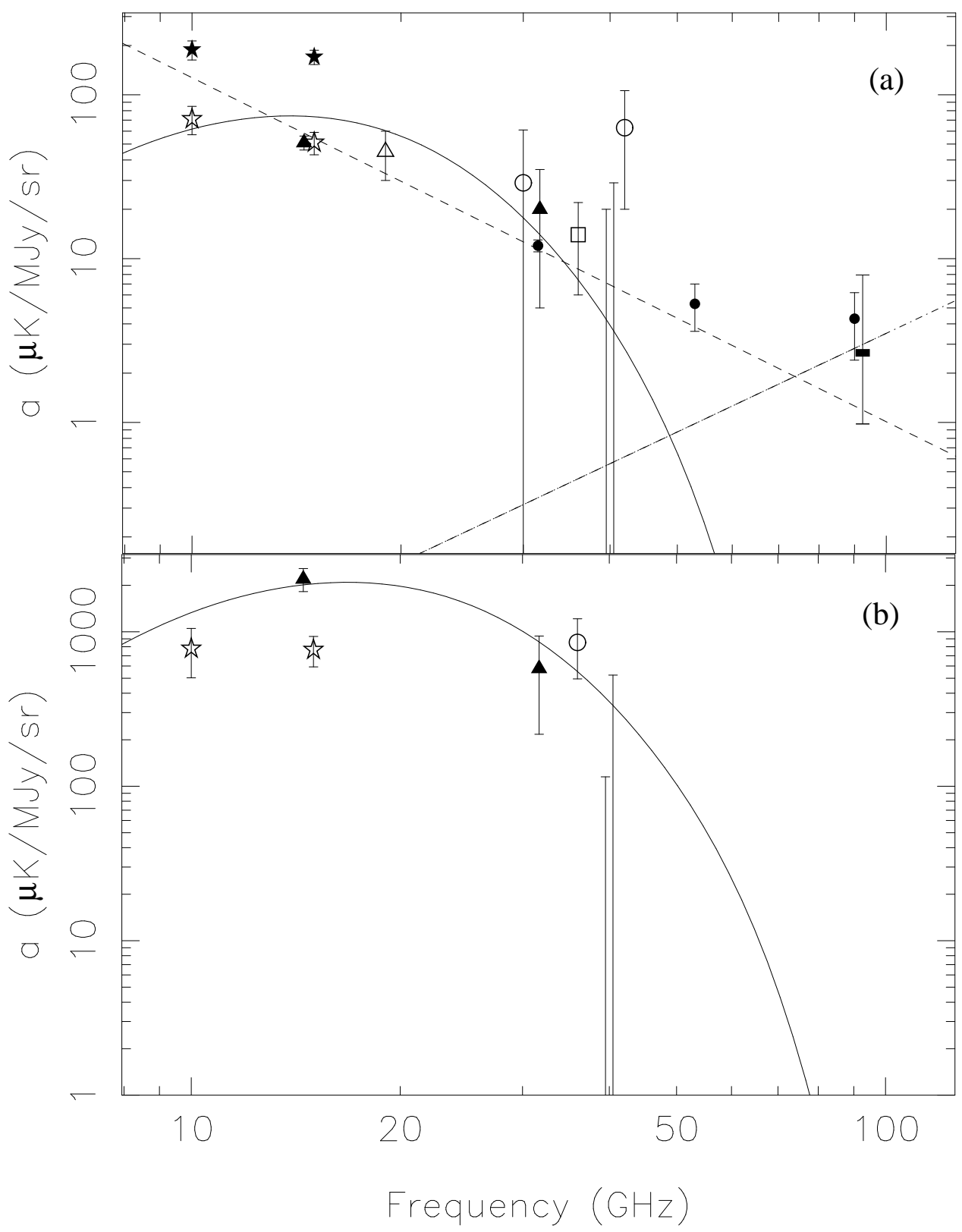

Fig. 1.- - a) Correlation slopes between CMB anisotropy data and the dust $100 \mu \mathrm{m}$ template. One standard deviation upper limits obtained for $\mathrm{PyV}$ from analysing all the fields and from analysing the SP94 overlap region only are both shown at $\sim 40 \mathrm{GHz}$. b) Correlations with the $12 \mu \mathrm{m}$ template. Results are also shown for other experiments: Tenerife (open stars, de Oliveira-Costa et al. 2001; filled stars, Mukherjee et al. 2001), OVRO (filled triangles), 19GHz (open triangles), SP94 (open circles), Saskatoon (open square), Boomerang (filled rectangle), and DMR (filled circles). The solid curves are representative of a spinning dust spectrum, the dashed and the dot-dashed lines represent free-free and vibrational dust emission spectra, respectively. 